\title{
Bond breaking in vibrationally excited methane on transition metal catalysts
}

\author{
R. Milot and A. P. J. Jansen \\ Schuit Institute of Catalysis, ST/SKA, Eindhoven University of Technology \\ P.O. Box 513, NL-5600 MB Eindhoven, The Netherlands
}

(October 24, 2018)

\begin{abstract}
The role of vibrational excitation of a single mode in the scattering of methane is studied by wave packet simulations of oriented $\mathrm{CH}_{4}$ and $\mathrm{CD}_{4}$ molecules from a flat surface. All nine internal vibrations are included. In the translational energy range from 32 up to $128 \mathrm{~kJ} / \mathrm{mol}$ we find that initial vibrational excitations enhance the transfer of translational energy towards vibrational energy and increase the accessibility of the entrance channel for dissociation. Our simulations predict that initial vibrational excitations of the asymmetrical stretch $\left(\nu_{3}\right)$ and especially the symmetrical stretch $\left(\nu_{1}\right)$ modes will give the highest enhancement of the dissociation probability of methane.
\end{abstract}

The dissociative adsorption of methane on transition metals is an important reaction in catalysis; it is the rate limiting step in steam reforming to produce syngas, and it is prototypical for catalytic C-H activation. Although the reaction mechanism has been studied intensively, it is not been fully understood yet. A number of molecular beam experiments in which the dissociation energy was measured as a function of translational energy have observed that vibrationally hot $\mathrm{CH}_{4}$ dissociates more readily than cold $\mathrm{CH}_{4}$, with the energy in the internal vibrations being about as effective as the translational energy in inducing dissociation. 6 Two independent bulbgas experiment with laser excitation of the $\nu_{3}$ asymmetrical stretch and $2 \nu_{4}$ umbrella modes on the $\operatorname{Rh}(111)$ surface, 8 and laser excitation of the $\nu_{3}$ and $2 \nu_{3}$ modes on thin films of rhodium $\theta$ did not reveal any noticeable enhancement in the reactivity of $\mathrm{CH}_{4}$. A recent molecular beam experiment with laser excitation of the $\nu_{3}$ mode did succeed in measuring a strong enhancement of the dissociation on a $\mathrm{Ni}(100)$ surface. However, this enhancement was still much too low to account for the vibrational activation observed in previous stydies and indicated that other vibrationally excited modes contribute significantly to the reactivity of thermal samples.10

Wave packet simulations of the methanedissociation reaction on transition metals have treated the methane molecule always as a diatomic up to now.11 16 Apart from one $\mathrm{C}-\mathrm{H}$ bond (a pseudo $\nu_{3}$ stretch mode) and the molecule surface distance, either (multiple) rotations or some lattice motion were included. None of them have looked at the role of the other internal vibrations, so there is no model that describes which vibrationally excited mode might be responsible for the experimental observed vibrational activation. In previous papers we have reported on wave packet simulations to determine which and to what extent internalvibrations are important for the dissociation of $\mathrm{CH}_{4}$ in the vibrational ground state, 17 and the isotope effect of $\mathrm{CD}_{4} .18$ We were not able yet to simulate the dissociation including all internal vibrations. Instead we simulated the scattering of methane, for which all internal vibrations can be included, and used the results to deduce consequences for the dissociation. These simulations indicate that for methane to dissociate the interaction of the molecule with the surface should lead to an elongated equilibrium $\mathrm{C}-\mathrm{H}$ bond length close to the surface. In this letter we report on new wave packet simulations of the role of vibrational excitations for the scattering of $\mathrm{CH}_{4}$ and $\mathrm{CD}_{4}$ molecules with all nine internal vibrations. The dynamical features of these simulations give new insight into the initial steps of the dissociation process. The conventional explanation is that vibrations help dissociation by adding energy needed to overcome the dissociation barrier. We find that two other new explanations play also a role. One of them is the enhanced transfer of translational energy into the dissociation channel by initial vibrational excitations. The other more important explanation is the increased accessibility of the entrance channel for dissociation.

We have wed the multi-configurational time-dependent Hartree (MCTDH) method for our wave packet simulation.19.20 This method can deal with a large number of degrees of freedom and with large grids. (See Ref. 21 for a recent review.) Initial translational energy has been chosen in the range of 32 to $128 \mathrm{~kJ} / \mathrm{mol}$. The initial state has been written as a product state of ten functions; one for the normally incident translational coordinate, and one for each internal vibration. All vibrations were taken to be in the ground state except one which was put in the first excited state. The orientation of the $\mathrm{CH}_{4} / \mathrm{CD}_{4}$ was fixed, and the vibrationally excited state had $a_{1}$ symmetry in the symmetry group of the molecule plus surface $\left(\mathrm{C}_{3 v}\right.$ when one or three $\mathrm{H} / \mathrm{D}$ atoms point towards the surface, and $\mathrm{C}_{2 v}$ when two point towards the surface.) The potential-energy surface is characterised by an elongation of the $\mathrm{C}-\mathrm{H}$ bonds when the molecule approaches the surfaces, no surface corrugation, and a molecule-surface part appropriate for $\mathrm{Ni}(111)$. It has been shown to give reasonable results, and is described in Refs. 17 and 18. These articles give also the computational details about the configurational basis and number of grid points, and contain illustrations of the orientations and the important vibrational modes.

We can obtain a good idea about the overall activation of a mode by looking at the kinetic energy expectation 
values $\left\langle\Psi(t)\left|T_{j}\right| \Psi(t)\right\rangle$ for each mode $j$. During the scattering process the change in the translational kinetic energy is the largest. It is plotted in Fig. 1 as a function of time for $\mathrm{CH}_{4}$ in the orientation with three bond pointing towards the surface with an initial kinetic energy of $96 \mathrm{~kJ} / \mathrm{mol}$ and different initial vibrational states. When the molecule approaches the surface the kinetic energy falls down to a minimum value. This minimum value varies only slightly with the initial vibrational states of the molecule. The total loss of translational kinetic energy varies substantially, however. The initial translational kinetic energy is not conserved. This means that the vibrational excitation enhances inelastic scattering. Especially an excitation of the $\nu_{1}$ symmetrical stretch and to a lesser extend the $\nu_{3}$ asymmetrical stretch mode result in an increased transfer of kinetic energy towards the intramolecular vibrational energy. The inelastic scatter component (the initial minus the final translational energy) for both isotopes in the orientation with three bonds pointing towards the surface, shows the following trend for the initial vibrational excitations of the modes; $\nu_{1}>\nu_{3}>\nu_{4}>$ ground state. $\mathrm{CH}_{4}$ scatters more inelastic than $\mathrm{CD}_{4}$ over the whole calculated range of translational kinetic energies, if the molecule has an initial excitation of the $\nu_{3}$ stretch mode. $\mathrm{CH}_{4}$ scatters also more inelastically than $\mathrm{CD}_{4}$ in the $\nu_{1}$ symmetrical stretch mode at higher energies, but at lower energies it scatters slightly less inelastically. For the molecules with the non-excited state or an excitation in the $\nu_{4}$ umbrella mode $\mathrm{CD}_{4}$ has a higher inelastic scattering component than $\mathrm{CH}_{4}$. At an initial translational kinetic energy of $128 \mathrm{~kJ} / \mathrm{mol}$ the excitation of the $\nu_{4}$ umbrella mode results in a strong enhancement of the inelastic scattering component. For $\mathrm{CD}_{4}$ the inelastic scattering component for the initial excited $\nu_{4}$ umbrella mode can become even larger than for the initial excited $\nu_{3}$ stretch mode. For the orientation with two bonds pointing towards the surface we observe the same trends for the relation between the inelastic scatter components and the excited initial vibrational modes, but the inelastic scatter component are less than half of the values for the orientation with three bonds pointing towards the surface. Also the excitation of the $\nu_{3}$ asymmetrical stretch modes results now in a higher inelastic scattering component for $\mathrm{CD}_{4}$ than for $\mathrm{CH}_{4}$. Excitation of the $\nu_{2}$ bending mode gives a little higher inelastic scatter component than the vibrational ground state. For the orientation with one bond pointing towards the surface we observe an even lower inelastic scattering component. At an initial kinetic energy of $128 \mathrm{~kJ} / \mathrm{mol}$ we find that both the $\nu_{1}$ and $\nu_{3}$ stretch modes have on inelastic component of around $6.5 \mathrm{~kJ} / \mathrm{mol}$ for $\mathrm{CD}_{4}$ and $4.0 \mathrm{~kJ} / \mathrm{mol}$ for $\mathrm{CH}_{4}$. At an initial translational energy of $32 \mathrm{~kJ} / \mathrm{mol}$ we observe for both isotopes in all orientations a very small increase of translational kinetic energy for the vibrational excited molecule, which means that there is a net transfer from intramolecular vibrational energy through the surface repulsion into the translational coordinate.

There seem to be two groups of vibrations with different qualitative behavior with respect to (de)excitation when the molecule hits the surface. The first group, let's call it the "stretch" group, consist of the $\nu_{3}$ asymmetric stretch in any orientation and the $\nu_{1}$ symmetric stretch in the orientation with three hydrogen/deuterium atoms pointing to the surface. The second, let's call it the "bending" group, consists of all bending vibrations and the $\nu_{1}$ in other orientations. When the molecule is initially in the vibrational ground state the kinetic energy in the vibrations increases, reaches a maximum at the turn-around point, and then drops back almost to the initial level except for a small contribution due to the inelastic scattering component. The vibrations within a group have very similar amounts of kinetic energy, but the "stretch" group has clearly a larger inelastic component than the "bending" group, and also the kinetic energy at the turn-around point is larger. When the molecule has initially an excitation of a vibration of the "stretch" group then the kinetic energy of that vibration increases, reaches a maximum at the turn-around point, and drops to a level lower than it was initially. For an excitation of a vibration of the "bending" group there is no maximum, but its kinetic energy simply drops to a lower level. We see that in all cases there is not only a transfer of energy from the translation to vibrations, but also an energy flow from the initially excited vibration to other vibrations. However, the total energy of the vibrational kinetic energy and the intramolecular potential energy increases, because it has to absorb the inelastic scattering component.

Figure 2 shows the (repulsive) interaction with the surface during the scattering process of $\mathrm{CH}_{4}$ at an initial kinetic energy of $96 \mathrm{~kJ} / \mathrm{mol}$ and different initial vibrational excitations for the orientation with three hydrogens pointing towards the surface. Since this is a repulsive term with a exponential fall-off changes in the repulsion indicate the motion of the part of the wave packet closest to the surface. At the beginning of the simulation the curves are almost linear in a logarithmic plot, because the repulsion hardly changes the velocity of the molecule. After some time the molecule enters into a region with a higher surface repulsion and the slopes of the curves drop. This results in a maximum at the turn-around point, where most of the initial translational kinetic energy is transfered into potential energy of the surface repulsion. For a classical simulation it would have meant no translational kinetic energy, but it corresponds with the minimum kinetic energy for our wave packet simulations. Past the maximum, a part of the wave packet will accelerate away from the surface, and the slope becomes negative. The expectation value of the translational kinetic energy (see Fig.1) increases at the same time. The slope of the curves in Fig. 2 becomes less negative towards the end of the simulation, although the expectation value of the translational kinetic energy in this time region is almost constant. The reason for this is that a part of the wave packet with less translational kinetic energy is still in a region close to the surface. We see also that the height of the plateaus for the different initial vibrational excitations is again in the order; $\nu_{1}>\nu_{3}>\nu_{4}>$ ground state. This again indicates that a larger part of 
the wave packet is inelastically scattered when $\nu_{1}$ is excited than when $\nu_{3}$ is excited, etc.

At lower initial translational kinetic energies the plateaus have a lower position and the main gap exist between the plateaus of the $\nu_{1}$ and $\nu_{3}$ stretch modes and the lower positioned plateaus of the $\nu_{4}$ umbrella and the ground state. At an initial translational kinetic energies of $128 \mathrm{~kJ} / \mathrm{mol}$ the positions of the plateaus are higher and the differences between the initial vibrational excitations are also smaller. The plateau of the $\nu_{3}$ stretch mode is even around the same position as the $\nu_{4}$ umbrella mode for $\mathrm{CD}_{4}$ in the orientation with three bonds pointing towards the surface at this initial energy. The orientation with two bonds pointing towards the surface shows the same trends. The plateaus of the initial excited $\nu_{2}$ bending mode are located slightly above the ground state for both isotopes. For the orientation with one bond pointing towards the surface the relative positions of the plateaus of the different initial excitations are the same as at low energies in the orientation with three bonds pointing towards the surface.

Even though we did not try to describe the dissociation itself, the scattering simulation do yield indications for the role of vibrational excitations on the dissociation of methane, and compare these with experimental observations. The dissociation of methane occurs over a late barrier, because it is enhanced by vibrational energy.22 Conventionally, the role of vibrational excitation on the enhancement of dissociation probability was discussed as an effect of the availability of the extra (vibrational kinetic) energy for overcoming the dissociation barrier. Our simulations show that such a process might play a role, but they show also that two other processes occur through vibrational excitation.

Firstly, an initial vibrational excitation increases translational kinetic energy transfer towards the intramolecular vibrational energy. The simulations show that this inelastic scatter component can be seen in an large enhancement of the vibrational kinetic energy in the stretch modes at the turn-around point. This increase is larger for higher initial translational kinetic energy and is most effective in the orientation with three bonds pointing towards the surface. If the dissociation of methane occurs primarily in this orientation, then we would expect, based on the total available vibrational energy after hitting the surface, that excitation of the $\nu_{1}$ symmetrical stretch mode is the most effective for enhancing the dissociation probability. The $\nu_{3}$ asymmetrical stretch mode appears to be less so. An explanation of the enhanced inelastic scatter compound by vibrational excitation is that through excitation the bonds are weakened, which will ease excitation in the initial non-excited modes. Other excitations than the $\nu_{2}, \nu_{3}$, or $\nu_{4}$ with $a_{1}$ symmetry for a particular orientation can possibly result in higher energy transfers, but we think that the difference with $\nu_{1}$ (which has always $a_{1}$ symmetry) would be still large.

Secondly, the accessibility of the dissociation channel enhances also the dissociation probability. We have concluded previously that our potential mimics reasonably the entrance channel for dissociation.17 In this letter we find that a part of the wave packet has a longer residence time at the surface. It is this part of the wave packet that accesses the dissociation channel, and it is also this part that is able to come near to the transition state for dissociation. From Figs. 1 and 2 we conclude that the $\nu_{1}$ stretch mode will enhance the dissociation probability the most. The enhanced accessibility by vibrational excitation is explained by the spread of the wave packet along a $\mathrm{C}-\mathrm{H}$ bond, which gives a higher probability for the system to be atop the dissociation barrier.

The molecular beam experiment with excitations of the $\nu_{3}$ asymmetrical stretch mode of $\mathrm{CH}_{4}$ of Ref. 10 shows that a single excitation of the $\nu_{3}$ asymmetrical stretch mode enhances dissociation, but the measured reactivity of the $\nu_{3}$ stretch mode is too low to account for the total vibrational activation observed in the molecular beam study of Ref. 5 . It means that excitation of another mode than the $\nu_{3}$ stretch will be more effective for dissociation. Our simulations show that indeed excitation of $\nu_{3}$ stretch will enhance dissociation, but predict that excitation of the $\nu_{1}$ symmetrical stretch mode will be more effective if the dissociation occurs primary in the orientation with multiple bonds pointing towards the surface. The contribution of the $\nu_{1}$ symmetrical stretch mode cannot be measured directly, because it has no infra-red activity. However, the contribution of the $\nu_{1}$ mode can be estimated using a molecular beam study as follows. The contribution of the $\nu_{3}$ stretch has already been determined.10 Similarly the contribution of the $\nu_{4}$ umbrella mode can be determined. The contribution of the $\nu_{2}$ bending can be estimated from our simulations to be somewhat lower than the $\nu_{4}$ umbrella contribution. The total contribution of all vibrations is known from Ref. 战, and a simple subtraction will give us then the contribution of the $\nu_{1}$ stretch. At high translational energies the accessibility of the dissociation channel for molecules with an excited $\nu_{4}$ umbrella mode is near to that of the molecules with excited stretch modes, and for $\mathrm{CD}_{4}$ the inelastic scattering is also enhanced. So the excitation of the $\nu_{4}$ umbrella mode can still contribute significantly to the vibrational activation, because it has also higher Boltzmann population in the molecular beam than the stretch modes.

This research has been financially supported by the Council for Chemical Sciences of the Netherlands Organization for Scientific Research (CW-NWO), and has been performed under the auspices of the Netherlands Institute for Catalysis Research (NIOK). 
${ }^{1}$ C. T. Rettner, H. E. Pfnür, and D. J. Auerbach, Phys. Rev. Lett. 54, 2716 (1985).

${ }^{2}$ C. T. Rettner, H. E. Pfnür, and D. J. Auerbach, J. Chem. Phys. 84, 4163 (1986).

${ }^{3}$ M. B. Lee, Q. Y. Yang, and S. T. Ceyer, J. Chem. Phys. 87, 2724 (1987).

${ }^{4}$ A. C. Luntz and D. S. Bethune, J. Chem. Phys. 90, 1274 (1989).

${ }^{5}$ P. M. Holmbad, J. Wambach, and I. Chorkendorff, J. Chem. Phys. 102, 8255 (1995).

${ }^{6}$ J. H. Larsen, P. M. Holmblad, and I. Chorkendorff, J. Chem. Phys. 110, 2637 (1999).

${ }^{7}$ A. V. Walker and D. A. King, Phys. Rev. Lett. 82, 5156 (1999).

${ }^{8}$ J. T. Yates, Jr., J. J. Zinck, S. Sheard, and W. H. Weinberg, J. Chem. Phys. 70, 2266 (1979).

${ }^{9}$ S. B. Brass, D. A. Reed, and G. Ehrlich, J. Chem. Phys. 70, 5244 (1979).

${ }^{10}$ L. B. F. Juurlink, P. R. McCabe, R. R. Smith, C. L. DiCologero, and A. L. Utz, Phys. Rev. Lett. 83, 868 (1999).

11 J. Harris, J. Simon, A. C. Luntz, C. B. Mullins, and C. T. Rettner, Phys. Rev. Lett. 67, 652 (1991).

12 A. C. Luntz and J. Harris, Surf. Sci. 258, 397 (1991).

13 A. C. Luntz and J. Harris, J. Vac. Sci. A 10, 2292 (1992).

14 A. C. Luntz, J. Chem. Phys. 102, 8264 (1995).

${ }^{15}$ A. P. J. Jansen and H. Burghgraef, Surf. Sci. 344, 149 (1995).

${ }^{16}$ M.-N. Carré and B. Jackson, J. Chem. Phys. 108, 3722 (1998).

${ }^{17}$ R. Milot and A. P. J. Jansen, J. Chem. Phys. 109, 1966 (1998).

${ }^{18}$ R. Milot and A. P. J. Jansen, Surf. Sci. (2000), to be published, arXiv:physics.chem-ph/0003031.

${ }^{19}$ U. Manthe, H.-D. Meyer, and L. S. Cederbaum, J. Chem. Phys. 97, 3199 (1992).

${ }^{20}$ A. P. J. Jansen, J. Chem. Phys. 99, 4055 (1993).

${ }^{21}$ M. H. Beck, A. Jäckle, G. A. Worth, and H.-D. Meyer, Phys. Rep. 324, 1 (2000).

${ }^{22}$ R. D. Levine and R. B. Bernstein, Molecular Reaction Dynamics and Chemical Reactivity (Oxford University Press, Oxford, 1987).

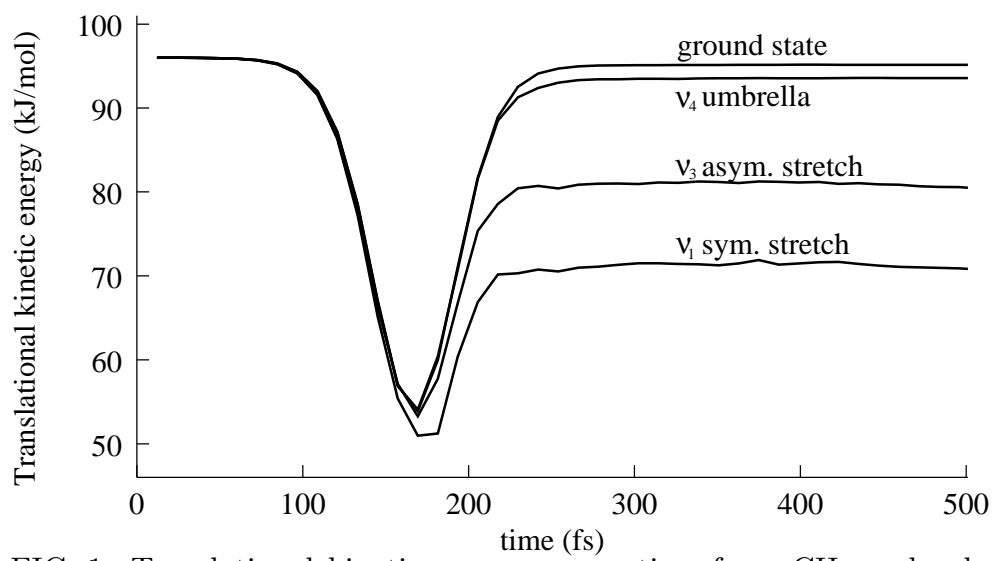

FIG. 1. Translational kinetic energy versus time for a $\mathrm{CH}_{4}$ molecule with three bonds pointing towards the surface. The initial translational kinetic energy is $96 \mathrm{~kJ} / \mathrm{mol}$.

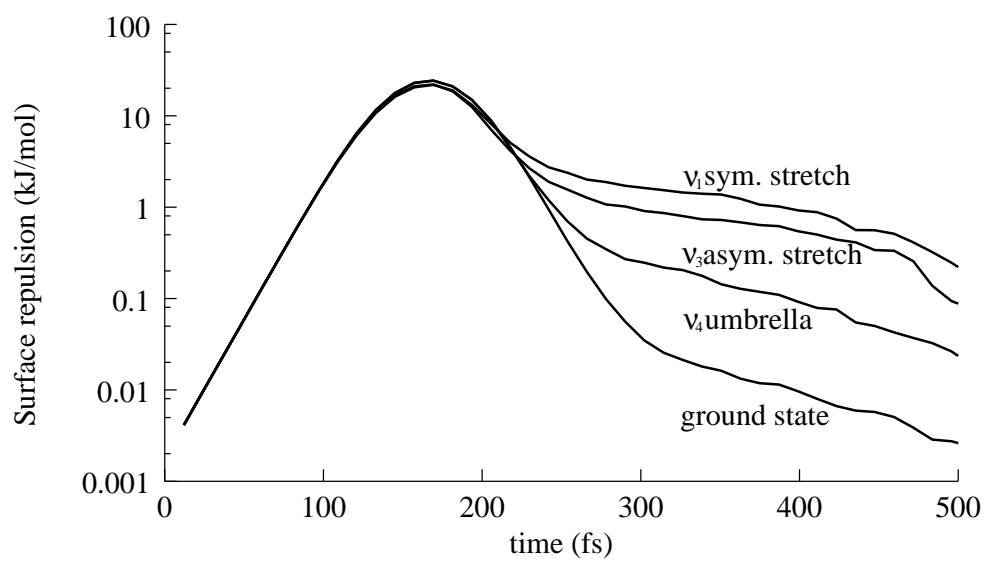

FIG. 2. Surface repulsion versus time during the scattering dynamics of $\mathrm{CH}_{4}$ at an initial translational energy of $96 \mathrm{~kJ} / \mathrm{mol}$ in the orientation with three bonds pointing towards the surface. 